\title{
PRIORIDADE OBSERVADA A PARTIR DA PRESUNÇÃO DE ATITUDE GAUSSIANA DAS ALTERNATIVAS (PrOPPAGA): PROPOSTA AXIOMÁTICA E DESENVOLVIMENTO DE UMA PLATAFORMA COMPUTACIONAL PARA UM NOVO MÉTODO MULTICRITÉRIO DE APOIO À TOMADA DE DECISÃO
}

\author{
Felipe Barbosa dos Santos (IME) Felipe.barbosa@ime.eb.br \\ Marcos dos Santos (IME) marcosdossantos_doutorado_uff@yahoo.com.br
}

\section{Resumo}

Os métodos de Apoio Multicritério a Decisão (AMD) buscam apoiar o tomador de decisão na escolha da alternativa mais preferível entre as várias possíveis, considerando os critérios que caracterizam essa preferência. No entanto, esta tarefa pode-se tornar muita complexa, dependendo do método utilizado. Isto ocorre porque os algoritmos dos métodos nem sempre são de simples aplicação. Desta forma, se torna indispensável o desenvolvimento de ferramentas computacionais que apliquem os algoritmos dos métodos de AMD, tornando viável a utilização dos métodos. Neste contexto, foi desenvolvida a ferramenta computacional apresentada neste artigo. Ela utiliza o método "Prioridade Observada a Partir da Presunção de Atitude Gaussiana das Alternativas" (PrOPPAGA) em um problema de seleção de classe de Navio de Assistência Hospitalar (NAsH) da Marinha do Brasil para o enfrentamento da pandemia do COVID-19. A principal contribuição deste artigo é apresentar um tutorial para utilização desta ferramenta, de forma a tornar viável a sua utilização pela sociedade.

Palavras-Chaves: AMD; COVID-19; Ferramenta computacional; Marinha do Brasil; PrOPPAGA

\section{Introdução}

Os métodos de Apoio Multicritério a Decisão (AMD) buscam apoiar o tomador de decisão na escolha da alternativa mais preferível entre as várias possíveis, considerando os critérios que caracterizam essa preferência. Problemas multicritérios podem ser divididos em contínuos, como programação linear, ou discretos (Kodikara 2008). Dentro de problemas discretos, alguns métodos definem o conceito de relação de indiferença $\left(a_{i} I a_{j}\right)$ e relação de preferência $\left(a_{i} P a_{j}\right)$ 
entre alternativas. Outros acrescentam os conceitos de incomparabilidade entre alternativas, preferência fraca e preferência estrita de uma alternativa em relação a outra (Bana e Costa \& Vincke 1990), utilizando, para sua aplicação, seus respectivos limiares de indiferença e preferência.

Os métodos AMD também são influenciados pela natureza e características dos dados usados. A natureza dos dados está intimamente ligada à escala de medição. Os dados podem ser quantitativos ou qualitativos e podem ser expressos na escala cardinal (quantitativa) ou ordinal (qualitativa) (Saaty 1980). O ser humano é, portanto, obrigado a tomar decisões, ou a partir de parâmetros quantitativos, ou a partir de parâmetros de medição qualitativos, com forte característica subjetiva. Os parâmetros quantitativos são geralmente mais fáceis de medir do que os parâmetros qualitativos. Por outro lado, as características dos dados referem-se ao fato de os dados serem corretos ou incertos (Öztürk et al. 2005). Atualmente, novos métodos, baseados na Teoria dos Conjuntos Fuzzy, expressam essas incertezas dos dados nebulosos (Tian et al. 2020; Dong 2021; Rudnik 2021; Ji et al. 2021; Tang et al. 2021; Song et al. 2021; Zarei et al. 2021; Chen \& Pan 2021; Karagöz et al. 2021; Liu et al. 2021).

Nesse contexto, saber estruturar o processo de tomada de decisão torna-se imprescindível (Barrager 2016). Com isto, a Marinha do Brasil vem adotando AMD para estruturar diversas decisões estratégicas da Força (Moreira et al. 2020, 2021; Tenório et al. 2020).

Via de regra, em problemas multicritério, quanto mais simples a ferramenta analítica, melhor (Raiffa 2002). Partindo dessa premissa, buscar modelagem com simplificações torna-se salutar, e entender o comportamento das alternativas auxilia nessa tarefa. A simplificação proposta nesta abordagem é a presunção de que as alternativas possuem comportamento gaussiano, ou seja, os dados das alternativas (amostra de valores), em cada um dos critérios considerados, apresentam um histograma em forma de sino (curva normal). A partir disso, os dados das alternativas são normalizados, tendo como referência a média e o desvio padrão da amostra.

Cinelli et al., (2020) enfatizam que especialmente os métodos mais recentes, normalmente mais avançados e permitindo avaliações mais complexas, por não possuírem um software que viabilize uma implementação trivial, prejudicam-se quanto a suas aplicabilidades em várias áreas. Com isso, podemos concluir que a utilização de determinado método, está ligado, muitas das vezes, a disponibilização de um software que permita a implementação do método a um dado caso. A principal contribuição deste artigo é apresentar uma ferramenta computacional 
para aplicação do método "Prioridade Observada a Partir da Presunção de Atitude Gaussiana das Alternativas" (PrOPPAGA), tornando viável a utilização do método pela sociedade.

O restante deste artigo está organizado da seguinte forma. A seção 2 apresenta o método PrOPPAGA. Na seção 3 é apresentada a ferramenta desenvolvida através de um estudo de caso para aplicação do método. Na seção 4 é realizada a conclusão.

\section{Método PrOPPAGA}

O método PrOPPAGA foi desenvolvido com o intuito de ser simples e eficaz comparado aos métodos consagrados na literatura, especificamente em problemas com muitas alternativas (mais de 30), onde alguns métodos que realizam comparações paritárias se tornam exaustivos para o decisor, e ainda podem incorrer em inconsistências, que geram retrabalho.

De acordo com Rezaei, (2015), um problema AMD é normalmente apresentado de acordo com a matriz da equação (1).

$$
P=\begin{gathered}
c_{1} \\
a_{1} \\
a_{2} \\
\vdots \\
a_{m}
\end{gathered}\left(\begin{array}{cccc}
p_{11} & p_{12} & \ldots & c_{n} \\
p_{21} & p_{22} & \ldots & p_{2 n} \\
\vdots & \vdots & \ddots & \vdots \\
p_{m 1} & p_{m 2} & \ldots & p_{m n}
\end{array}\right)
$$

Onde $\left\{a_{1}, a_{2}, \ldots, a_{m}\right\}$ é o conjunto de alternativas analisadas, $\left\{c_{1}, c_{2}, \ldots, c_{n}\right\}$ é o conjunto de critérios considerados, e $p_{i j}$ é a pontuação obtida pela alternativa $a_{i}$ no critério $c_{j}$. O objetivo é selecionar a alternativa com o maio valor geral. O valor geral $v_{i}$, da alternativa $a_{i}$, é calculado através de (2), assumindo-se um peso $w_{j}$ para o critério $c_{j}\left(w_{j} \geq 0, \sum w_{j}=1\right)$.

$$
v_{i}=\sum_{j=1}^{n} w_{j} \cdot p_{i j}
$$

Na próxima seção, veremos que o PrOPPAGA utiliza a mesma matriz (1) e a equação (2) no seu passo-a-passo.

\subsection{Passo-a-passo PrOPPAGA}

Nesta seção, será apresentado o passo-a-passo do método. 


\subsubsection{Passo 1. Determinar o conjunto de critérios de decisão}

Nesta etapa é definido o conjunto $C=\left\{c_{1}, c_{2}, \ldots, c_{n}\right\}$, que será utilizado para a tomada de cisão. Para critérios quantitativos é importante observar quais são monotônicos de custo. Em critérios monotônicos de custo, as alternativas terão os valores dos parâmetros multiplicados por (-1) para expressar o impacto negativo destes valores.

\subsubsection{Passo 2. Ordenar os critérios por importância}

Uma vez definido $C$, os critérios devem ser ordenados por ordem de importância (Figura 1), podendo, inclusive, serem equiparados.

Figura 1 - Exemplo de ordenação com cinco critérios

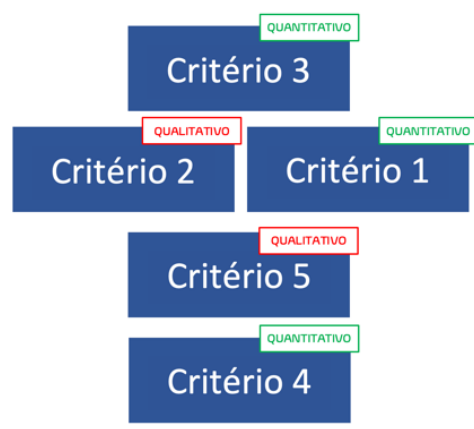

Fonte: Autores (2021)

\subsubsection{Passo 3. Atribuir grau de importância $\left(s_{j}\right)$ e pesos $\left(w_{j}\right)$ dos critérios}

De acordo com o ordenamento realizado no passo anterior, um grau de importância $s_{j}$ será atribuído a cada critério $c_{j}$. O valor máximo de $s_{j}$ obedece a relação (3).

$$
\left(s_{j}\right)_{\text {max }}= \begin{cases}n, & \text { se } n>7 \\ 7, & \text { se } n \leq 7\end{cases}
$$

Onde $n$ é o número de elementos do conjunto $C$. Ao(s) critério(s) mais importante(s), deve ser atribuído $\left(s_{j}\right)_{\max }$. Aos demais critérios, será atribuído um grau de importância menor, de acordo com o ordenamento feito, como podemos observar no exemplo da Figura 2. 
Figura 2 - Exemplo de ordenação, com cinco critérios

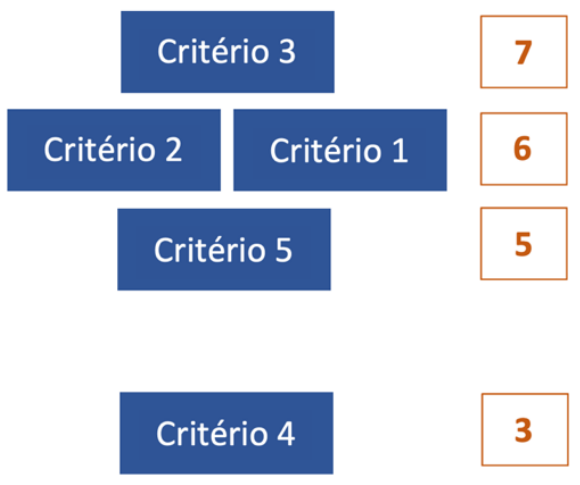

Fonte: Autores (2021)

$W$ é definido como o conjunto de pesos dos critérios, onde $W=\left\{w_{1}, w_{2}, \ldots, w_{j}\right\}$ e $w_{j}$ o peso do critério $c_{j}$. A partir da média ponderada (4), obtemos o valor de $w_{j}$.

$$
w_{j}=\frac{s_{j}}{\sum_{j=1}^{n} s_{j}}
$$

Com isso, $\sum w j=1$, como pode ser observado no exemplo da Figura 3.

Figura 3 - Exemplo de cálculo de peso dos critérios

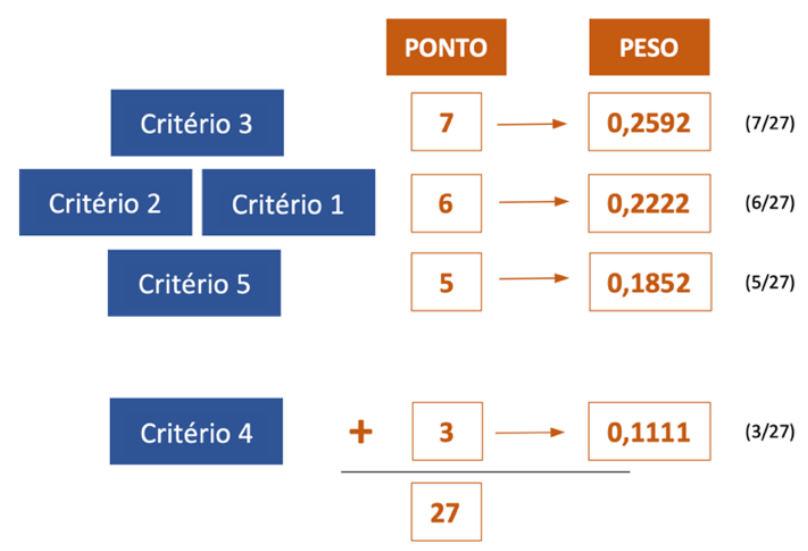

Fonte: Autores (2021)

\subsubsection{Passo 4. Tomar as alternativas}

É definido $A=\left\{a_{1}, a_{2}, \ldots, a_{m}\right\}$ como o conjunto de alternativas analisadas para a tomada decisão. Em cada critério, as alternativas apresentam um atributo $d_{i j}$. Estes atributos formam a Matriz de Decisão $M$. 


$$
M=\left[\begin{array}{ccc}
d_{11} & \cdots & d_{1 n} \\
\vdots & \ddots & \vdots \\
d_{m 1} & \cdots & d_{m n}
\end{array}\right]
$$

Espera-se que os atributos dos critérios quantitativos tenham seus valores bem definidos, atrelados à uma unidade de medida. No entanto, é importante observar os critérios monotônicos de custo, pois nestes critérios, os atributos serão representados por valores negativos. Para critérios qualitativos, onde não é possível utilizar uma unidade de medida, os atributos serão definidos por uma escala de sete pontos, onde a performance de cada alternativa é avaliada de acordo com a Tabela 1 .

Tabela 1 - Escala de sete pontos do PrOPPAGA

\begin{tabular}{cl}
\hline Pontuação & \multicolumn{1}{c}{ Definição } \\
\hline 1 & A alternativa não atende as demandas \\
2 & A alternativa atende as demandas bem abaixo do esperado \\
3 & A alternativa atende as demandas um pouco abaixo do esperado \\
4 & A alternativa atende as demandas dentro do esperado \\
5 & A alternativa atende as demandas um pouco acima das expectativas \\
6 & A alternativa atende as demandas bem acima das expectativas \\
7 & A alternativa supera todas as expectativasem relação a este critério \\
\hline
\end{tabular}

Fonte: Autores (2021)

\subsubsection{Passo 5. Normalização}

Uma vez definidos os atributos, seus valores serão normalizados baseado na presunção de que eles se comportam de forma Gaussiana dentro de cada critério. Então, para cada critério $c_{j} \in C$, a média $\mu_{\mathrm{j}}$ e o desvio padrão $\sigma_{j}$, dos atributos $d_{i j}$, são calculados.

A probabilidade $P$ de um atributo qualquer $x$ ser menor ou igual que $d_{i j}$, representa o elemento $p_{i j}$ da Matriz de Decisão Normalizada $N$.

$$
N=\left[\begin{array}{ccc}
p_{11} & \cdots & p_{1 n} \\
\vdots & \ddots & \vdots \\
p_{m 1} & \cdots & p_{m n}
\end{array}\right]
$$

Segundo Albuquerque et al., (2008),

$$
p_{i j}=P\left(x \leq d_{i j}\right)=\int_{-\infty}^{d_{i j}} \frac{1}{\sqrt{2 \pi} \sigma_{j}} e^{-\frac{\left(x-\mu_{j}\right)^{2}}{2 \sigma_{j}^{2}}} d x
$$

Graficamente, $p_{i j}$ é a área abaixo da curva Gaussiana (definida pela média $\mu_{\mathrm{j}}$ e pelo desvio 
padrão $\sigma_{j}$ ), limitada à direita por $d_{i j}$ (Figura 4).

Figura 4 - Representação gráfica de $p_{i j}$

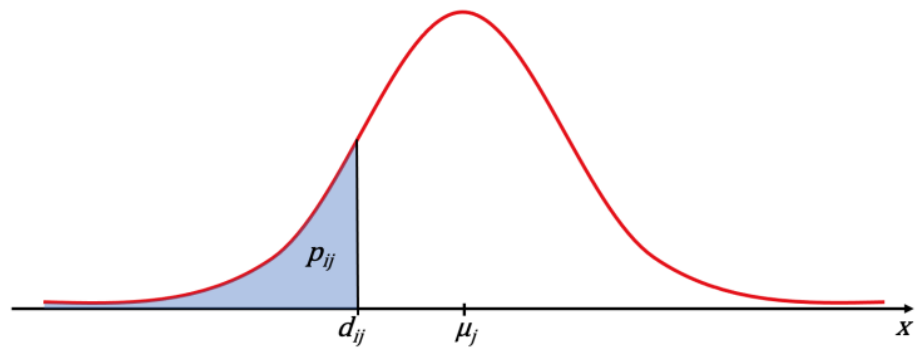

Fonte: Autores (2021)

\subsubsection{Passo 6. Agregação}

O valor geral $v_{i}$ é calculado pela equação (2), onde $v_{i}$ representa a cardinalidade da $i$-ésima alternativa. Uma outra forma de interpretar esta agregação é através da relação $N \cdot w=v$, ou ainda:

$$
\left[\begin{array}{ccc}
p_{11} & \cdots & p_{1 n} \\
\vdots & \ddots & \vdots \\
p_{m 1} & \cdots & p_{m n}
\end{array}\right] \cdot\left[\begin{array}{c}
w_{1} \\
\vdots \\
w_{n}
\end{array}\right]=\left[\begin{array}{c}
v_{1} \\
\vdots \\
v_{m}
\end{array}\right]
$$

\section{Ferramenta computacional}

Um método de AMD, em essência, é um algoritmo que quando aplicado, retorna um resultado para os dados inseridos nele. No entanto, os cálculos/etapas que são realizados ao longo deste processo nem sempre são triviais, ou mesmo, de domínio de quem o emprega. Desta forma, o desenvolvimento de ferramentas computacionais possibilita que um usuário que não domine o algoritmo de um determinado método possa utilizá-lo, sem incorrer em erros

conceituais, uma vez que a interatividade com o usuário faz com que o mesmo seja guiado durante todo o processo de emprego do método. Neste contexto, mesmo que o algoritmo de uma determinada abordagem seja relativamente simples, o desenvolvimento de uma ferramenta computacional se torna essencial para disseminação deste método AMD na sociedade.

A ferramenta computacional desenvolvida para aplicação do método PrOPPAGA pode ser acessada no sítio eletrônico www.proppaga.com.br. Ela foi desenvolvida em linguagem PHP, através da IDE Apache NetBeans 12.2. A fim de apresentar a ferramenta, será mostrado uma aplicação dela em um problema real, de forma a se observar o passo-a-passo a ser seguido. 


\subsection{Estudo de caso}

A fim de apresentar a ferramenta computacional desenvolvida para o método PrOPPAGA, ela será utilizada na ordenação de quatro Navios Hospitalares da Marinha do Brasil para apoiar o combate à pandemia de covid-19. O problema foi exposto por Costa et al., (2020), que aplicou o método THOR 2 para solucioná-lo.

Diante do cenário de pandemia de covid-19, os sistemas de saúde de diversas localidades do país foram levados ao limite. Neste contexto, a Marinha do Brasil surge como um aliado no enfrentamento à pandemia. A Marinha do Brasil dispõe de Navios de Assistência Hospitalar (NAsH) sediados na região Norte e Centro-Oeste do país. Estes navios desempenham a função de hospitais flutuantes que, normalmente, já operam na região amazônica, levando apoio de saúde às comunidades ribeirinhas.

No navio selecionado seria implantado um Hospital de Campanha (HCAMP) para atender pacientes acometidos de doenças não contagiosas, a fim de desonerar os hospitais de Manaus e cidades próximas, ou mesmo de cidades mais distantes, dependendo do agravamento da pandemia na região.

As classes de Navio analisadas foram: "Oswaldo Cruz", "Dr. Montenegro", "Soares Meirelles" e "Tenente Maximiano". Os critérios utilizados para a tomada de decisão foram:

Manobrabilidade: Capacidade do navio se deslocar ao adentrar e atracar em portos com pouca profundidade, comuns nas regiões ribeirinhas. Este é um critério qualitativo.

Tripulação (unidade): Quantidade de pessoal embarcado para operar o navio. Uma tripulação numerosa diminui o conforto a bordo. Entende-se que quanto menor a tripulação, melhor.

Raio de ação (milhas náuticas): Distância máxima que o navio consegue alcançar, saindo de sua sede e a ela retornando, sem necessidade de reabastecimento.

Velocidade Máxima (nós): Critério auto explicativo, onde quanto maior a velocidade máxima, melhor.

Capacidade de evacuação de pacientes: Outro critério qualitativo. É levado em consideração as possibilidades de meios para a transferência de pacientes, por razões de ordem médica, para outras unidades de saúde.

Capacidade hospitalar: Considera os tipos de atendimentos médicos possíveis, bem como a quantidade de leitos disponíveis e a possibilidade de uma eventual ampliação deste número, por meio da instalação de um HCAMP a bordo. 
O grau de importância atribuído à cada critério é mostrado na Tabela 2.

Tabela 2 - Grau de importância de cada critério

\begin{tabular}{rc}
\hline Critério & Grau de importância \\
\hline Manobrabilidade & 3 \\
Tripulação & 2 \\
Raio de ação & 3 \\
Velocidade Máxima & 6 \\
Capacidade de evacuação de pacientes & 7 \\
Capacidade hospitalar & 7 \\
\hline
\end{tabular}

Fonte: Autores (2021)

Os dados operativos dos Navios analisados podem ser observados na Tabela 3. Estes dados serão utilizados, futuramente, para definir a Matriz de Decisão.

Tabela 3 - Dados operativos dos Navios analisados

\begin{tabular}{|c|c|c|c|c|c|}
\hline Critério & Característica & $\begin{array}{l}\text { NAsH Dr. } \\
\text { Montenegro }\end{array}$ & $\begin{array}{c}\text { NAsH } \\
\text { Oswaldo } \\
\text { Cruz }\end{array}$ & $\begin{array}{l}\text { NAsH Soares } \\
\text { Meirelles }\end{array}$ & $\begin{array}{c}\text { NAsH Tenente } \\
\text { Maximiano }\end{array}$ \\
\hline \multirow{4}{*}{ Manobrabilidade } & Comprimento & 42 & 47,2 & 63 & 31,06 \\
\hline & Largura (m) & 11 & 8,45 & 12 & 6,5 \\
\hline & Calado (m) & 2,4 & 1,75 & 2,1 & 1,02 \\
\hline & $\begin{array}{l}\text { Deslocamento } \\
\text { Máximo (ton) }\end{array}$ & 347 & 490 & 1338 & 160 \\
\hline Tripulação & $\begin{array}{l}\text { Número de } \\
\text { pessoas }\end{array}$ & 60 & 27 & 47 & 23 \\
\hline Raio de ação & $\begin{array}{l}\text { Em milhas } \\
\text { náuticas }\end{array}$ & 3.200 & 3.000 & 6.000 & 1.100 \\
\hline $\begin{array}{l}\text { Velocidade } \\
\text { Máxima }\end{array}$ & Em nós & 10 & 12 & 12 & 12 \\
\hline $\begin{array}{l}\text { Capacidade de } \\
\text { evacuação de } \\
\text { pacientes }\end{array}$ & $\begin{array}{c}\text { Recursos de } \\
\text { evacuação de } \\
\text { pacientes }\end{array}$ & $\begin{array}{c}2 \text { lanchas para } \\
\text { transporte de } \\
\text { pessoal }\end{array}$ & $\begin{array}{c}\text { Convoo capaz } \\
\text { de operar um } \\
\text { helicóptero } \\
\text { Bell Jet } \\
\text { Ranger IH-6 } \\
\text { ou Esquilo } \\
\text { UH-12, além } \\
\text { de } 2 \text { lanchas } \\
\text { para } \\
\text { transporte de } \\
\text { pessoal }\end{array}$ & $\begin{array}{c}2 \text { lanchas para } \\
\text { transporte de } \\
\text { pessoal }\end{array}$ & $\begin{array}{l}2 \text { lanchas para } \\
\text { transporte de } \\
\text { pessoal }\end{array}$ \\
\hline
\end{tabular}




\begin{tabular}{|c|c|c|c|c|c|}
\hline & $\begin{array}{c}\text { Quantidade de } \\
\text { leitos } \\
\text { hospitalares } \\
\text { disponíveis }\end{array}$ & 6 & 6 & 6 & 3 \\
\hline $\begin{array}{l}\text { Capacidade } \\
\text { hospitalar }\end{array}$ & $\begin{array}{l}\text { Atendimentos } \\
\text { médicos } \\
\text { disponíveis }\end{array}$ & $\begin{array}{l}3 \text { consultórios, } \\
2 \text { gabinetes } \\
\text { odontológicos, } \\
1 \text { laboratório, } \\
1 \text { farmácia, } 1 \\
\text { sala de raio X, } \\
2 \text { enfermarias, } \\
1 \text { sala de } \\
\text { cirurgia, } 1 \text { sala } \\
\text { de } \\
\text { emergência, } \\
\text { UTI }\end{array}$ & $\begin{array}{c}2 \\
\text { ambulatórios, } \\
2 \text { gabinetes } \\
\text { odontológicos, } \\
1 \text { laboratório, } \\
1 \text { farmácia, } 1 \\
\text { sala de raio X, } \\
2 \text { enfermarias, } \\
1 \text { sala de } \\
\text { cirurgia }\end{array}$ & $\begin{array}{l}\text { Consultórios } \\
\text { médicos, } \\
\text { odontológicos, } \\
\text { farmácia, sala } \\
\text { de vacinação, } \\
\text { sala } \\
\text { de raio X, } \\
\text { centro } \\
\text { cirúrgico, } \\
\text { enfermaria, } \\
\text { laboratório de } \\
\text { análises } \\
\text { clínicas }\end{array}$ & $\begin{array}{l}\text { Centro cirúrgico, } \\
\text { enfermaria, sala de } \\
\text { esterilização, sala } \\
\text { de expurgo, } \\
\text { farmácia, } \\
\text { laboratório, } \\
\text { consultório médico, } \\
\text { consultórios } \\
\text { odontológicos, } 1 \\
\text { compartimento } \\
\text { equipado com } \\
\text { aparelho de raio-X }\end{array}$ \\
\hline
\end{tabular}

Fonte: Autores (2021)

Para utilização da ferramenta computacional do PrOPPAGA é necessário, após acessar o endereço eletrônico, informar que decisão precisa ser tomada (Figura 5) e clicar em "PRÓXIMO".

Figura 5 - Qual decisão precisa ser tomada

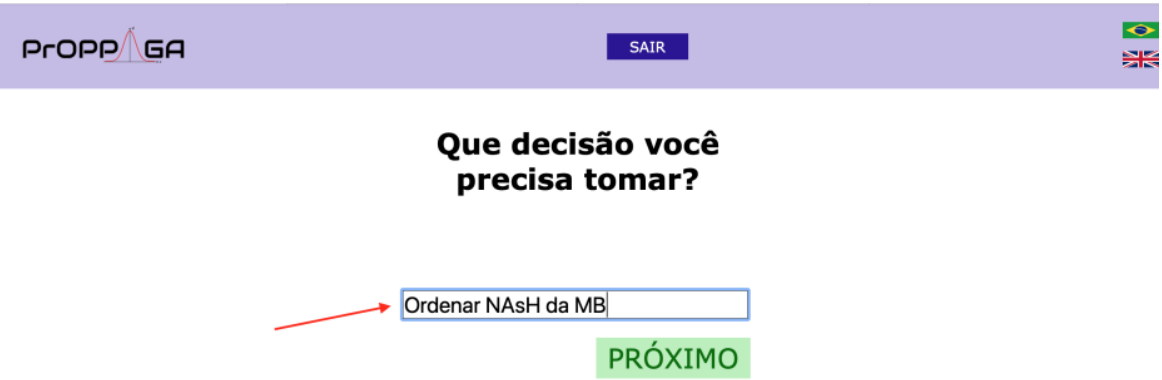

Fonte: www.proppaga.com.br

Então será solicitado que informe quantos critérios serão utilizados para esta tomada de decisão (Figura 6). 
Figura 6 - Quantos critérios serão utilizados

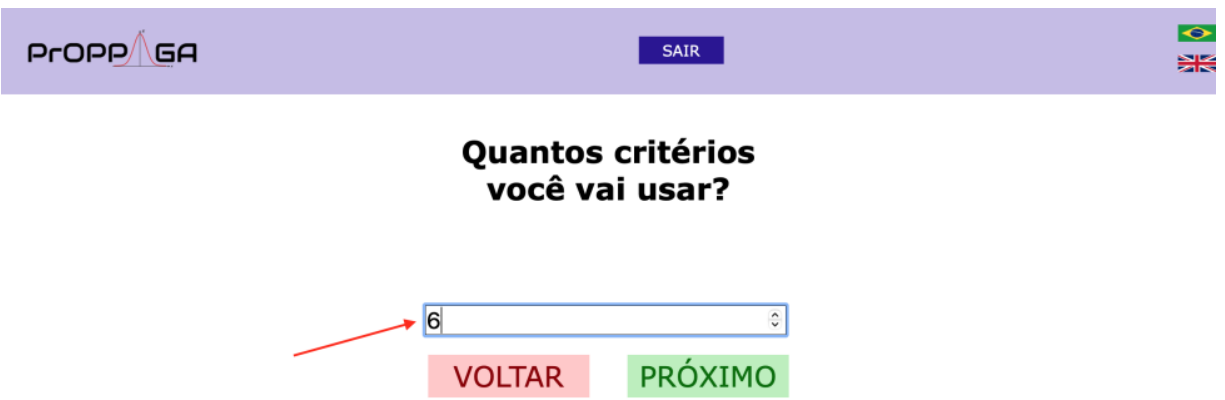

Fonte: Autores (2021)

Depois disso, será necessário informar os critérios utilizados (Figura 7). Cada critério informado deverá ser classificado em “QUANTITATIVO” ou “QUALITATIVO”, dependendo da natureza do critério. Para critérios classificados como "QUANTITATIVO”, ainda será solicitado que seja informado se os valores buscados em cada um destes critérios são os "Máximos" ou "Mínimos". Critérios monotônicos de custo buscam os valores mínimos, enquanto que, critérios monotônicos de lucro buscam os valores máximos.

Figura 7 - Critérios utilizados

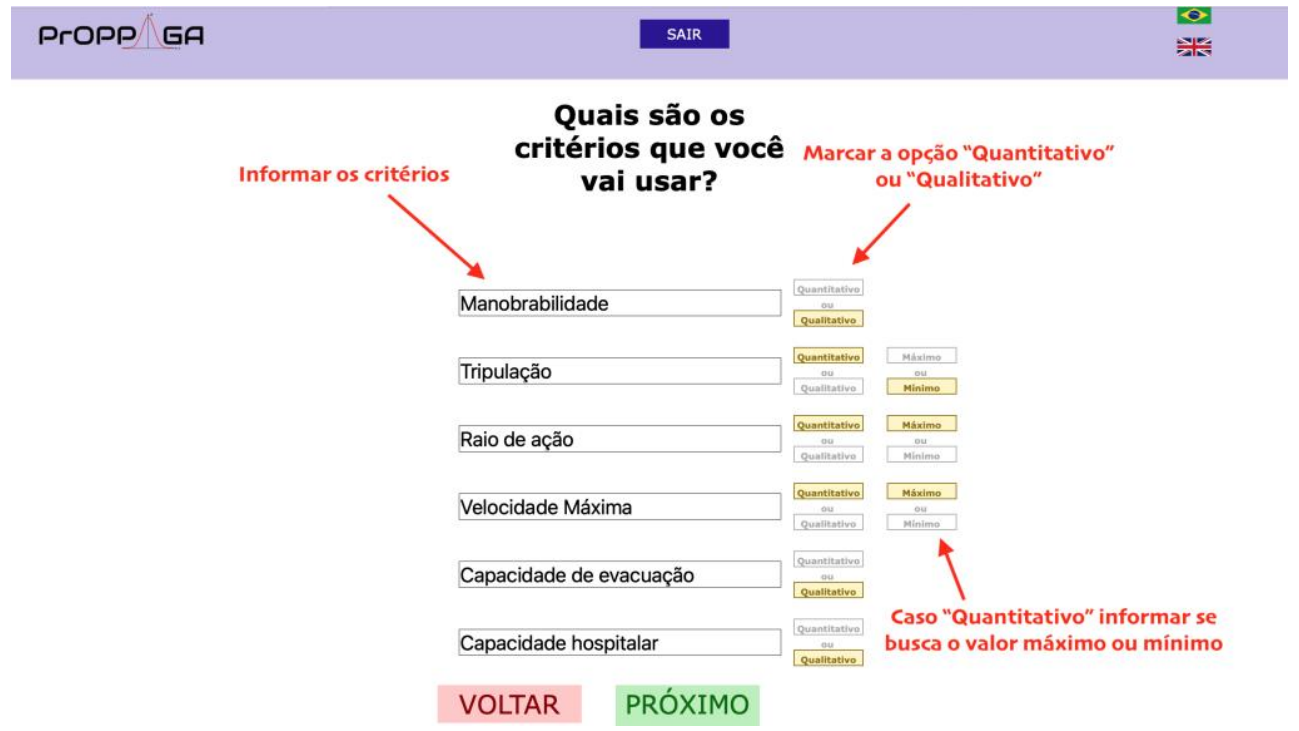

Fonte: www.proppaga.com.br

O próximo passo é a definição do grau de importância de cada critério (Figura 8). Aqui foram inseridas as informações de acordo com a Tabela 2. 
Figura 8 - Grau de importância de cada critério

\begin{tabular}{|c|c|c|c|}
\hline PrOPP & GA & SAIR & 잉 \\
\hline
\end{tabular}

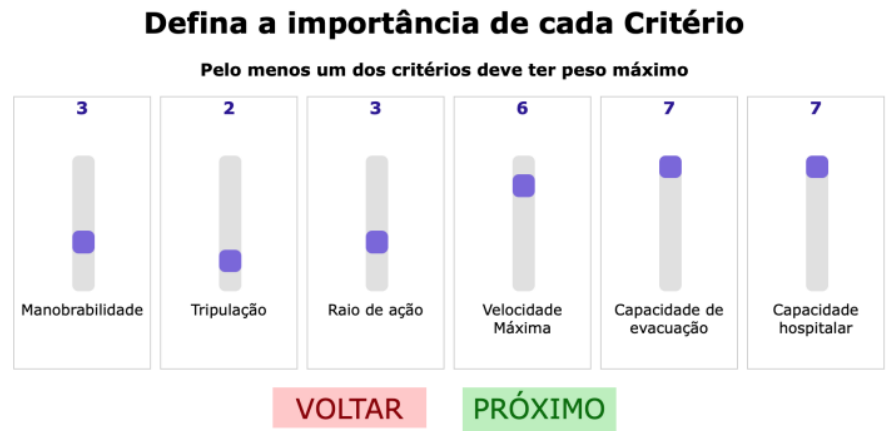

Fonte: www.proppaga.com.br

Com o grau de importância dos critérios definidos, a própria ferramenta calcula o peso de cada critério. No entanto, esta informação só será exibida para o usuário na última etapa, que é a exibição do resultado.

A próxima etapa é a adição de alternativas (Figura 9). As alternativas são inseridas uma a uma. Importante observar que é solicitado nesta etapa os atributos dos critérios quantitativos. Após inserir as informações referente a uma alternativa deve se clicar no botão "+” para adicionar a alternativa à lista de alternativas.

Figura 9 - Adicionar alternativas

$\begin{array}{llll}\text { PrOpP GP } & \text { SAIR }\end{array}$

Adicionar Alternativas

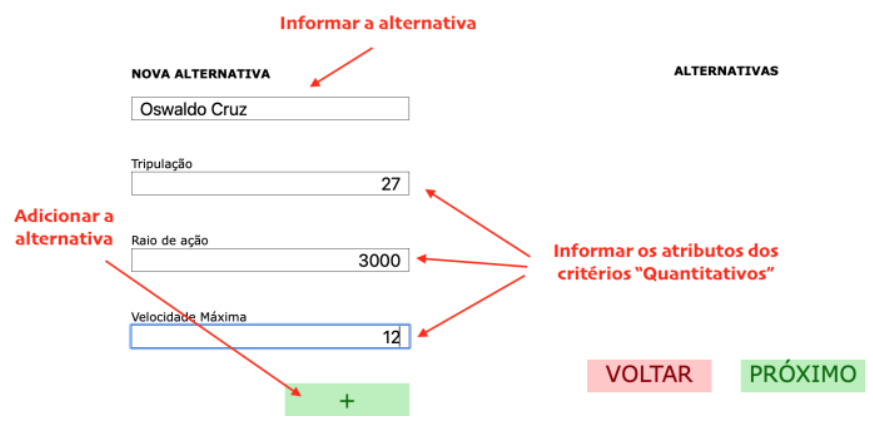

Fonte: www.proppaga.com.br 
As alternativas vão sendo adicionadas à lista de alternativas que fica à direita da tela. Após todas as alternativas serem inseridas, deve-se clicar em "PRÓXIMO" (Figura 10) para ir para a próxima etapa.

Figura 10 - Lista de alternativas completa

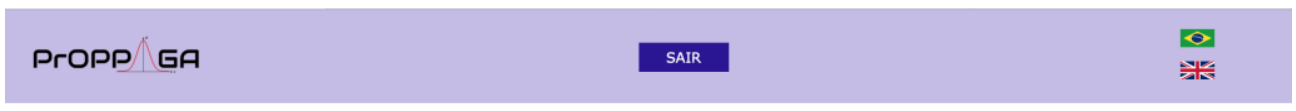

Adicionar Alternativas
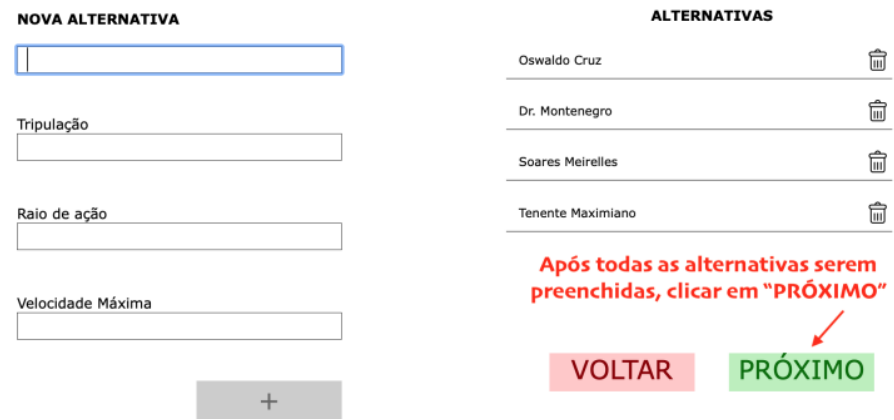

Após todas as alternativas serem preenchidas, clicar em "PRÓxIMO"

\section{VOLTAR PRÓXIMO}

Fonte: www.proppaga.com.br

Uma vez que todas as alternativas tenham sido inseridas, com as respectivas informações nos critérios quantitativos, será solicitado que seja feita a avaliação das alternativas nos critérios qualitativos (Figuras 11 e 12), de acordo com a escala de sete pontos (Tabela 1).

Figura 11 - Avaliação do critério qualitativo Manobrabilidade

PrOPP GP SAIR

Avalie as alternativas em cada critério

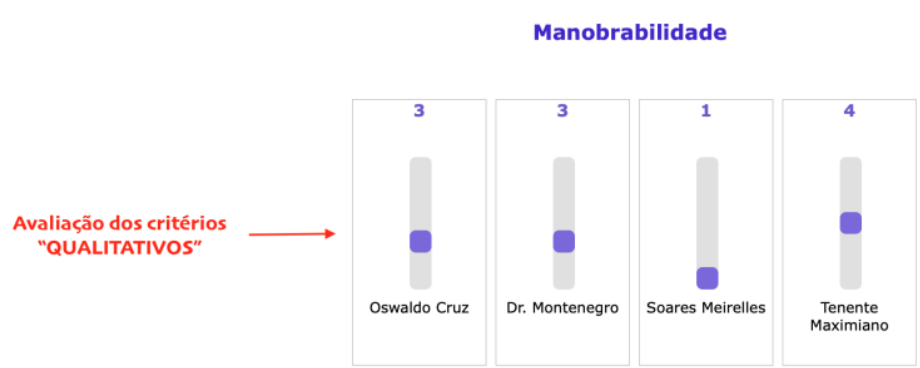

Fonte: www.proppaga.com.br 
Figura 12 - Avaliação dos critérios qualitativos Capacidade de evacuação e Capacidade hospitalar

Capacidade de evacuação

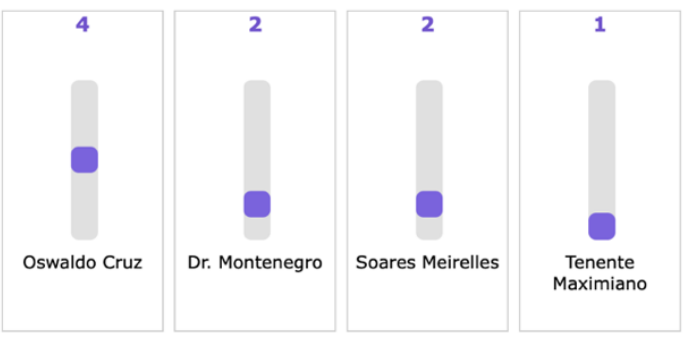

Capacidade hospitalar

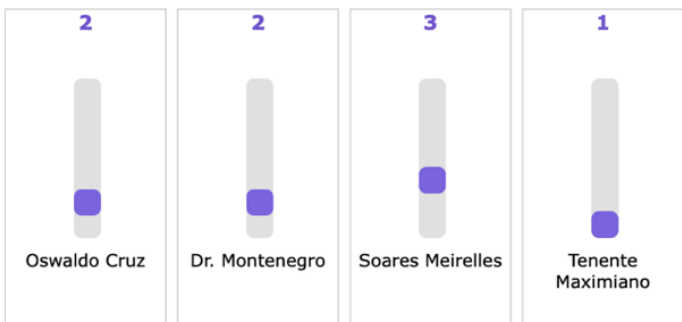

\section{VOLTAR PRÓXIMO}

Fonte: www.proppaga.com.br

Os critérios são exibidos em sequência. Abaixo do último critério, aparece o botão "PRÓXIMO", que deve ser acionado após o preenchimento de todos os critérios. Então será exibido o resultado encontrado (Figura 13), onde além deste resultado, são exibidos também todos os valores utilizados pelo método PrOPPAGA durante o processo.

Figura 13 - Resultado do PrOPPAGA

\begin{tabular}{|c|c|c|c|c|c|c|c|}
\hline PrOPP EA & & & SAIR & & & & 은 \\
\hline \multicolumn{8}{|c|}{$\begin{array}{l}\text { RESULTADOS PARA } \\
\text { Ordenar NAsH da MB }\end{array}$} \\
\hline CRITÉRIO & $\begin{array}{c}\text { Capacidade } \\
\text { dea } \\
\text { evacuarão }\end{array}$ & $\begin{array}{c}\text { Capacidade } \\
\text { hospitalar }\end{array}$ & $\begin{array}{l}\text { Velocidade } \\
\text { Máxima }\end{array}$ & Manobrabilidade & $\begin{array}{c}\text { Raio de } \\
\text { ação }\end{array}$ & Tripulação & \\
\hline $\begin{array}{l}\text { NOTAS } \\
\text { PESO }\end{array}$ & $\begin{array}{c}\text { evacuaçao } \\
7,25 \\
0,25\end{array}$ & $\begin{array}{c}7 \\
0,25\end{array}$ & $\begin{array}{c}6 \\
0,21\end{array}$ & $\begin{array}{c}3 \\
0,11\end{array}$ & $\begin{array}{c}3 \\
0,11\end{array}$ & $\begin{array}{c}2 \\
0,07\end{array}$ & \\
\hline $\begin{array}{c}\text { MÉDIA } \\
\text { DESVIO PADRÃO }\end{array}$ & $\begin{array}{l}2,25 \\
1,09\end{array}$ & $\begin{array}{l}2,00 \\
0,71\end{array}$ & $\begin{array}{l}11,50 \\
0,87\end{array}$ & $\begin{array}{l}2,75 \\
1,09\end{array}$ & $\begin{array}{l}3.325,00 \\
1.748,39\end{array}$ & $\begin{array}{l}39,25 \\
15,04\end{array}$ & \\
\hline \multicolumn{8}{|l|}{ Valores Informados } \\
\hline $\begin{array}{l}\text { Oswaldo Cruz } \\
\text { Soares Merirelles } \\
\text { Tenente Maximiano } \\
\text { Dr. Montenegro }\end{array}$ & $\begin{array}{l}4 \\
2 \\
1 \\
2\end{array}$ & $\begin{array}{l}2 \\
3 \\
1 \\
2\end{array}$ & $\begin{array}{l}12 \\
12 \\
12 \\
10\end{array}$ & $\begin{array}{l}3 \\
1 \\
4 \\
3\end{array}$ & $\begin{array}{l}3000 \\
6000 \\
1100 \\
3200\end{array}$ & $\begin{array}{l}27 \\
47 \\
23 \\
60\end{array}$ & \\
\hline Notas por Critério & & & & & & & $\begin{array}{l}\text { Nota } \\
\text { Final }\end{array}$ \\
\hline $\begin{array}{l}\text { Oswaldo Cruz } \\
\text { Soares Meruelles } \\
\text { Tenente Maximiano } \\
\text { Dr. Montenegro }\end{array}$ & $\begin{array}{l}9,46 \\
4,09 \\
1,26 \\
4,09\end{array}$ & $\begin{array}{l}5,00 \\
9,21 \\
0,79 \\
5,00\end{array}$ & $\begin{array}{l}7,18 \\
7,18 \\
7,18 \\
0,42\end{array}$ & $\begin{array}{l}5,91 \\
0,54 \\
8,74 \\
5,91\end{array}$ & $\begin{array}{l}4,26 \\
9,37 \\
1,02 \\
4,72\end{array}$ & $\begin{array}{l}7,92 \\
3,03 \\
8,60 \\
0,84\end{array}$ & $\begin{array}{l}6,80 \\
6,14 \\
3,70 \\
3,56\end{array}$ \\
\hline \multicolumn{8}{|c|}{ VOLTAR } \\
\hline
\end{tabular}

Fonte:www.proppaga.com.br 
Corroborando o que fora proposto por Costa et al., (2020), através do método THOR 2, o resultado obtido pelo PrOPPAGA apresenta extamente a mesma ordenação das alternativas. Indicando que a melhor alternativa é o NAsH Oswaldo Cruz. A cardinalidade exposta pela ferramenta apresenta os valores encontrados pelo método PrOPPAGA multiplicado por 10, a fim de enfatizar as diferenças de valores gerais entre as alternativas. O resultado é exposto em ordem decrescente destes valores.

\section{Conclusão}

A ferramenta computacional apresentada neste artigo possibilita a tomadores de decisão aplicar o método PrOPPAGA para auxiliá-los em problemas complexos. O fato dela estar disponível na internet, possibilita ao usuário sua utilização a qualquer tempo, em qualquer lugar, sem necessidade de instalar nenhum arquivo para isso e sem limitações de sistemas operacionais. Tal disponibilidade, aliada a uma interface gráfica amigável e de fácil compreensão torna a utilização do método PrOPPAGA viável, mesmo para aqueles que desconhecem seu algoritmo. O PrOPPAGA se mostra eficaz, uma vez que seus resultados foram validados por comparação ao método THOR 2. O que também corrobora o trabalho de Costa et al., (2020), trazendo robustez para a proposta de indicação da classe NAsH Oswaldo Cruz para o enfrentamento da pandemia do covid-19.

Esta ferramenta não tem restrições de uso, podendo ser utilizada tanto no meio civil, como no meio militar. Também pode ser utilizada tanto no meio público, quanto no meio privado. Desta forma, entende-se que o objetivo deste artigo fora alcançado, disponibilizando para a sociedade uma ferramenta computacional para aplicação do método PrOPPAGA.

\section{Referências}

AlbuQuerque J.P., Fortes J.M. \& Finamore W. Probabilidade, Variáveis Aleatórias e Processos Estocásticos.Interciência, 2008

Bana e Costa C. \& Vincke P. Multiple Criteria Decision Aid: An Overview. Readings in Multiple Criteria Decision Aid.Springer Berlin Heidelberg, 1990

BARRAGer S.M. A new engineering profession is emerging: decision coach. IEEE Engineering Management Review 44 (2): 33-40, 2016 https://doi.org/10.1109/EMR.2016.2568765

CHEN L. \& PAN W. 2021. - Review fuzzy multi-criteria decision-making in construction management using a network approach. Applied Soft Computing 102: 107103. 
https://doi.org/10.1016/j.asoc.2021.107103

Cinelli M., KADZINSKi M., GonZAleZ M. \& SlowinsKi R. How to support the application of multiple criteria decision analysis? Let us start with a comprehensive taxonomy, 2020 Omega 96: 102261. https://doi.org/10.1016/j.omega.2020.102261

Costa I.P. DE A., MaÊda S.M. do N., TeIXEIRA L.F.H. DE S. DE B., GoMES C.F.S. \& SANTOS M.D. Choosing a hospital assistance ship to fight the covid-19 pandemic. Revista de Saúde Pública 54: 79, 2020. https://doi.org/10.11606/s1518-8787.2020054002792

DONG J. Fuzzy best-worst method based on triangular fuzzy numbers for multi-criteria decision-making. Information Sciences: 25, 2021

Ji Y., Xu Y., QU S., XU Z., Wu Z. \& NABE M. 2021. — A Novel Two-Stage Multi-Criteria

Decision-Making Method Based on Interval-Valued Pythagorean Fuzzy Aggregation Operators with Self-Confidence Levels. Arabian Journal for Science and Engineering 46 (2): 1561-1584. https://doi.org/10.1007/s13369-020-04681-6

KARAGÖz S., DEVECI M., SimIC V. \& AYDIN N. Interval type-2 Fuzzy ARAS method for recycling facility location problems, 2021. Applied Soft Computing 102: 107107. https://doi.org/10.1016/j.asoc.2021.107107

KodiKara P.N.Multi-Objective Optimal Operation of Urban Water Supply Systems Australia, Victoria University, 2008.

LIU P., ZHU B. \& WANG P. A weighting model based on best-worst method and its application for environmental performance evaluation, 2021 Applied Soft Computing 103: 107168. https://doi.org/10.1016/j.asoc.2021.107168

Moreira M.Â.L., De Araújo Costa I.P., Pereira M.T., Dos Santos M., Gomes C.F.S. \& Muradas F.M. PROMETHEE-SAPEVO-M1 a Hybrid Approach Based on Ordinal and Cardinal Inputs: Multi-Criteria Evaluation of Helicopters to Support Brazilian Navy Operations, 2021. Algorithms 14 (5): 140. https://doi.org/10.3390/a14050140

MoreIRA M.Â.L., Gomes C.F.S., Dos Santos M., Silva M. Do C. \& Araujo J.V.G.A. PROMETHEE-SAPEVO-M1 a Hybrid Modeling Proposal: Multicriteria Evaluation of Drones for Use in Naval Warfare, in THOMÉ A.M.T., BARBASTEFANO R.G., SCAVARDA L.F., Dos ReIs J.C.G. \& AMORIM M.P.C. (eds.), 2020, Cham. Springer International Publishing. p. 381-393. https://doi.org/10.1007/978-3-030-56920-4_31

ÖZTÜRK M., TsOUKIÀS A. \& VINCKE P.Preference modelling. Multiple criteria decision analysis: 45, 2005

RAIFFA H. Decision Analysis: A Personal Account of How It Got Started and Evolved. Operations Research 50 (1): 179-185, 2002. https://doi.org/10.1287/opre.50.1.179.17797

REZAEI J. Best-worst multi-criteria decision-making method. Omega 53: 49-57, 2015. https://doi.org/10.1016/j.omega.2014.11.009

RUDNIK K. Ordered fuzzy WASPAS method for selection of improvement projects. 
Expert Systems With Applications: 18, 2021

SaAty T.L. The Analytic Hierarchy Process: Planning, Priority Setting, Resource Allocation.McGraw-Hill International Book Company. (Advanced book program), 1980

SONG Y., THATCHER D., Li Q., McHuGH T. \& Wu P. Developing sustainable road infrastructure performance indicators using a model-driven fuzzy spatial multi-criteria decision making method. Renewable and Sustainable Energy Reviews 138: 110538, 2021. https://doi.org/10.1016/j.rser.2020.110538

TANG C., XU D. \& CHEN N. Sustainability prioritization of sewage sludge to energy scenarios with hybrid-data consideration: a fuzzy decision-making framework based on full consistency method and fusion ranking model. Environmental Science and Pollution Research 28 (5): 5548-5565, 2021. https://doi.org/10.1007/s11356-020-10544-2

Tenório F.M., Dos Santos M., Gomes C.F.S. \& Araujo J. DE C. Navy Warship Selection and Multicriteria Analysis: The THOR Method Supporting Decision Making, in Thomé A.M.T., BARbastefano R.G., ScAvarda L.F., Dos Reis J.C.G. \& AMORIM M.P.C. (eds.), 2020, Cham. Springer International Publishing. p. 27-39. https://doi.org/10.1007/9783-030-56920-4_3

Tian G., Hao N., Zhou M., Pedrycz W., Zhang C., Ma F. \& Li Z.Fuzzy Grey Choquet Integral for Evaluation of Multicriteria Decision Making Problems With Interactive and Qualitative Indices. IEEE Transactions on Systems, Man, and Cybernetics: Systems: 1-14, 2020. https://doi.org/10.1109/TSMC.2019.2906635

ZAREI E., RAMAVANDi B., DARABI A.H. \& OMIDVAR M. A framework for resilience assessment in process systems using a fuzzy hybrid MCDM model. Journal of Loss Prevention in the Process Industries 69: 104375, 2021

https://doi.org/10.1016/j.jlp.2020.104375 